\title{
An integrative view on entropic measures applied to the nervous
} system

\author{
Diego M. Mateos ${ }^{1,2}$ and Jose Luis Perez Velazquez ${ }^{3, *}$ \\ ${ }^{1}$ Facultad de Ciencia y Tecnología. Universidad Autónoma de Entre Ríos (UADER). Oro Verde, Entre Ríos, \\ Argentina. \\ ${ }^{2}$ Instituto de Matemática Aplicada del Litoral (IMAL-CONICET-UNL). Santa Fe, Argentina. \\ ${ }^{3}$ The Ronin Institute, Montclair, NJ, 07043 USA; \\ ${ }^{*}$ Corresponding author: Jose Luis Perez Velazquez, jose.velazquez@ronininstitute.org, jlpvjlpv@gmail.com.
}

\begin{abstract}
In the same manner that there are several statements of the second law of thermodynamics but all of them are equivalent, it is possible that most of the entropic methods applied to the nervous system and brain in particular share a similar outcome, or essence, that help understand some aspects of the structure of cognition. In this short review focused on certain aspects of the entropic metrics some results are examined that indicate the fundamental importance of the natural tendency towards a maximal energy distribution for brain activity to be optimal and thus cognition to emerge.
\end{abstract}

\section{Introduction}

Entropic measures have been applied to the nervous system in many shapes and forms. Considering the almost ethereal nature of entropy (didn't von Neumann advise Shannon to call his measure entropy because "nobody knows what entropy really is?") these various applications to a number of measured variables (ions, neuronal connections, neurophysiological signals, etc.) pose the risk of confusion, of misleading the interpretations and thus the research to understand nervous system function.

The purpose of this text is an attempt at clarification of what some entropic measures applied to experimentally obtained variables mean and at the same time to try to distill the essence of these applications, to find a common theme that improves the understanding of the nervous systems and perhaps glimpse at the emergence of cognition. Hence, we will not focus on very specific technical applications of entropic metrics to particular nervous system variables but rather the emphasis will be on the neurophysiological meaning of some studies that may reveal fundamentals of the basic structure of cognitive neurodynamics. Finally, these results here surveyed suggest that the common theme underlying many entropic measures revolves around the well-known tendency of natural phenomena towards a maximal energy distribution - nothing more than the second principle of thermodynamicswhich in terms of the nervous system is manifested by maximisation of the number of configurations of cellular contacts or connections.

\section{The many faces of entropy}

As aforementioned, entropy has been applied to a variety of variables recorded, or obtained, from the nervous systems. If Thurner et al. described the three faces of entropy in the analysis of complex systems [1, here we present a concise survey of some aspects of nervous system activity that have been used for diverse entropy methods; the main purpose being to exhibit the many applications and some results but without going deep into any of these (there are so many that an entire encyclopedia would be needed!).

Starting at the microscopic level, entropy has been applied to characterise time dependent signals in sequences of discrete, identical action potentials or spikes, information being carried in the spike arrival time and entropy is used in this context to quantify this information without any assumption about which feature of the spike train is more important 2]. Following this line of thought, other authors have studied the upper limit of the information content of a single spike using Landauer's principle, the results indicating that an action potential can process more information than a single bit of the Shannon entropy [3]. Entropy has been applied as well to the molecular world, e.g., a mathematical model for ion diffusion in ion channels studied aspects of ion diffusion contributing to entropy production [4. Synapses have not escaped either to the application of entropic metrics, for instance 
addressing the balance between excitatory and inhibitory synapses needed to ensure appropriate function of neural networks, study that demonstrated highest entropy at the boundary between excitation-dominant and inhibition-dominant regimes [5]; as well, correlation entropy has been applied to synaptic input-output dynamics showing that cortical synapses exhibit low-dimensional chaos driven by natural input patterns [6].

At the macroscopic scale, entropy calculations have been very widely used to analyze electroencephalographic (EEG) signals, be it scalp EEG or intracranial/intracerebral EEG, and of course magnetoencephalographic (MEG) recordings and functional neuroimaging (fMRI) signals. The vast majority of computations are based on Shannon entropy and differ about which method is used to discretize the signal, e.g. binarization, histograms or wavelets. A widely used measure is the so-called permutation entropy $(\mathrm{PE})$, which is a robust time series tool that provides a quantification of the complexity of a dynamic system capturing the order relations between values in the time series and then extracting a probability distribution of the ordinal patterns [7. The PE is based on Shannon entropy but others entropies have been used, such as Renyí or Tsalli's entropy [8, 9. It has had myriad applications in the analysis of signals ranging from epilepsy and anaesthesia to Alzheimer's disease [10-12. There are modifications of these methods that take into account the length of the signal [13] or that give weights to the amplitude of the signal [14.

Spectral entropy (SE) has been popular too in the analysis of neural signals at the collective level (those abovementioned EEG, MEG and similar recordings that record the activity of large cell ensembles). SE is a Shannon entropy measure over the spectral power distribution of the signal. It treats the signal's normalized power distribution in the frequency domain as a probability distribution, and calculates the Shannon entropy of it; the power frequency domain can be obtained using the Fourier [15], Hilbert [16] or wavelet transform (called Wavelet entropy) [17. A major, practical application is in the analysis of EEG signals under anaesthesia to assess the level of patient sedation; these studies have shown that as sedation increases the entropy values associated with the EEG signals decrease [15]. As well, SE has been used in the study of sleep stages [18] and in the detection of epileptiform activity [19. The wavelet entropy, which has been proposed as a metric to evaluate brain rhythms using event related potentials [20, has been applied to assess recovery after brain injury [21] and in Alzheimer's disease [22].

Functional neuroimaging data, such as fMRI, have been subject to entropy measurements in many studies. As illustrations, it has been used to characterize ageing properties of the human brain through the analysis of resting state blood oxygen level dependent (BOLD) signals, indicating that the functional entropy associated with brain activity increase with age 23]; as well, a link between human intelligence and brain entropy has been sought in a study that found that brain entropy was positively associated with intelligence [24, and in the abstract of the paper the authors mention that "This relationship between high brain entropy and high intelligence indicates an essential role for entropy in brain functioning". It may be worth pointing out now one aspect, perhaps a warning, about all these entropy estimations that will be addressed in following sections, namely, that in reality rather than entropy having a "role in" some event, it is the physical meaning of the measure -energy distribution, see section 4, its underlying physical substrate that has the role, because one should not forget that entropy is a measure we have devised to characterise natural phenomena, but whether it can be considered a cause of processes driving living systems is a matter of debate [25]. It will be emphasised in sections below that entropy is an index of energy dispersal [26] and thus energy gradients are the fundamental essence directing the evolution of natural phenomena. We close this brief overview of entropy applications to nervous system observables with a mention of studies using sample entropy applied to fMRI in subjects with attention deficit hyperactivity disorder, revealing abnormalities in various brain regions [27, and in patients with schizophrenia where more complex signal patterns were found in the patients as compared to healthy individuals [28].

\section{Entropy as an information metric: energetic considerations}

Entropy has been very widely employed as a metric of information. The matter of information to characterise nervous system function has been taken with great enthusiasm since times past, which is reasonable because, as W. Bialek mentioned: "The generation of physicists who turned to biological phenomena in the wake of quantum mechanics noted that, to understand life, one has to understand not just the flow of energy but also the flow of information. There is, of course, some difficulty in translating the colloquial notion of "information" into something mathematically precise." [29]. Indeed, brain information processing has been used many times in the literature in its widest, intuitive sense (for a general account of what is conceived by brain computation and information processing see [30]) and while it is true that the concept of information has several connotations and is somewhat relative, the notion of information derived from entropy is more or less well characterised and understood, thanks to the works of C. Shannon and others. It has been the Shannon framework that has been taken by several investigators to address questions about neural coding. Let us recall that in the Shannon formalism entropy should not be conceived in terms of disorder - as it is the usual but, as mentioned below, it is 
not quite correct and this will be a fundamental point in tour discussion- but rather as a measure on a probability distribution characterizing, so to speak, the amount of missing information that distribution represents.

Therefore, at a microscopic scale, the neuronal action potentials (spikes) have been treated in some works as binary electrochemical switches -1 when the neuron fires the spike and 0 when they are at rest [31 33. Since the probability that a neuron will fire an action potential is influenced by many factors such as firing threshold, temperature, presynaptic inputs, degree of connectivity and so forth, these two states can be treated as random variables $X_{\text {firing }}$ and $X_{\text {resting. }}$. The probability of occurrence for each one is represented as $P_{f}=P\left(X_{f}\right)$ and $P_{r}=P\left(X_{r}\right)$ respectively, satisfying $P_{f}+P_{r}=1$; we can then write $P_{r}=1-P_{f}$ and replacing in Shannon's formula $S=-\sum_{i} P_{i} \cdot \log \left(P_{i}\right)$ we obtain

$$
S=-P_{f} \cdot \log \left(P_{f}\right)-\left(1-P_{f}\right) \cdot \log \left(1-P_{f}\right)
$$

That is, the entropy of this system depends on the probability of the neuron firing. This framework has been widely applied to address the question of neural coding. For instance, to compare the information transferred in particular neurocomputational models of the stimulus-response function, showing that dynamic stimuli can be encoded efficiently by single neurons and that each spike contributes to information transmission 34.

Similar analysis could be made at a macroscale level defining the states of the system as the pairwise connections between brain areas (or, to be more precise, between brain signals recorded from the tissue) 35 . In this case, the random variable becomes the connectivity between two networks: "connected", $X_{c}$, or "disconnected", $X_{d c}$, with the associated probabilities $P_{c}$ and $P_{d c}$ respectively. Connections in these cases meaning functional connections, that is, any metric like a synchrony index that is high enough, above a certain threshold, to consider the two networks (signals) as "connected". As before, we can write the entropy in function of probabilities of connections

$$
S=-P_{c} \log \left(P_{c}\right)-\left(1-P_{c}\right) \log \left(1-P_{c}\right)
$$

In both cases the graphic of the entropy $S$ is an inverted $\mathrm{U}$, reaching the peak at $P_{f}=P_{r}=1 / 2$, or at the macroscale case at $P_{c}=P_{d c}=1 / 2$; This maximum in the curve represents the maximum uncertainty of the system, or, in the statistical mechanics framework, the maximization of the possible (micro)states of the system.

But it is the relation of the Shannon formalism with the energy distribution or dispersal that interests us here, because entropy is not really a measure of disorder or chaos as commonly taught, entropy should not be used to estimate the degree of disorder in self-organising systems [36. Entropy represents energy distribution. Therefore rather than asserting that entropy is a driving force, it could be more reasonable to assert that energy diffusion, the energy dissipation or dispersion in a final state compared to an initial state is the driving force, entropy just being the index of that dispersal within a system and between the system and its surroundings [26].

The relation between energy and entropy or information has been the subject of study for many decades [37; indeed it was Clausius' original intention to relate energy and entropy -thermal entropy being part of the total energy content measuring how much of that energy becomes useless. Many scholars have further contributed fundamental notions on the topic, e.g., the Landauer's principle connecting the information theoretic notion of entropy to statistical mechanics. Let us note in our context that in the same manner as the equiprobability of states maximise the Shannon entropy $S=-\sum_{i} P_{i} \cdot \log \left(P_{i}\right)$ the equal distribution of energy among the possible states maximise too that entropy. To see this is trivial: maximal entropy occurs for equiprobable events $P_{i}=1 / N$ (say, of a total $N$ states), then using Boltzmann's approach: $P_{i}=e^{-E_{i} / K_{B} T}$ and taking $k_{B} T=1$ and $P_{i}=1 / N$ then $1 / N=e^{-E_{i}}$ hence $E_{i}=\log (N)$ all states have same energy. Therefore increasing entropy entails increasing energy distribution among the microstates. One can as well substitute energy for probability in Shannon's formula yielding $S \sim \sum_{i}=e^{-E_{i}} \cdot E_{i}$ which serves to perform analyses relating directly the energy of the microstates to the entropy $S$, but this is of course beyond the scope of a review article. The important matter to discuss here is what this equal energy dispersal among the microstates may reveal or say about brain dynamics. The next section expounds the possible neurophysiological interpretation of this result in light of past experiments and analyses done on brain recordings.

\section{Entropic measures applied to the brain networks' collective ac- tivity: maximization of configurations of network connections for optimal brain function}

Let us consider now what entropy applied to the study of the brain coordination dynamics -the cellular collective activity at the meso or macroscale level- may reveal in terms of possible fundamental principles of brain organization from where cognition and conscious awareness emerge. The reason to emphasise the cellular collective dynamics to understand the integrative functions of nervous systems is exemplified in the words of the neurologist 
and polymath Oliver Sacks: "That the brain is minutely differentiated is clear: there are hundreds of tiny areas crucial for every aspect of perception and behavior... The miracle is how they all cooperate, are integrated together, in the creation of the world. This, indeed, is the problem, the ultimate question, in neuroscience..." 38. It is therefore important to explore at a relatively high level of description that cooperation among brain cellular ensembles, for it is known that each neuron's activity is meaningful only with respect to other cells' actions; the foundation of neural information processing lies in the interaction between cells, and this is the reason why so many studies are devoted to the assessment of the patterns of neuronal organized activity using metrics like synchrony, coherence, correlations and similar methods. Cognition is after all a pattern of organised activity in the (embodied) nervous system, paraphrasing E.J. Ambrose's words "Life is basically a pattern of organized activity" 39].

Results derived from the estimation of the entropy associated with the number of possible configurations of pairwise brain network connectivity indicate that brain macrostates associated with conscious awareness possess more microstates (in terms of configurations of network connections) and thus higher entropy than unconscious states [35, 40. These studies used invasive and non-invasive electrophysiological brain recordings in different states of consciousness (sleep, wakefulness, seizures and coma) and the phase synchrony between pairs of signals was evaluated, then a threshold value of the synchronization index was used to determine when two signals were "connected" (see section 4.1 for notes on the putative "connection" between brain networks/signals). Each state of consciousness studied was considered a macrostate of brain synchronization, composed of a number of microstates which are the several possible configurations of the connected signals.

The entropy applied to the distribution of cellular connectivity, neural synchronization and similar aspects are therefore related to the characterization of cellular interactions, how the activity of cell networks are organised, but activity is after all energy. Hence, the essence of the entropy applied to most of these variables detecting cellular activity is about energy distribution. Finding that entropy associated with the number of the configurations of brain networks is largest during states of conscious awareness [35, 40] is an indication that energy is maximally distributed among those networks in these behavioural states. Following thermodynamic considerations about the time evolution of the entropy, energy dissipation was found larger during conscious awareness as opposed to unconscious pathological states -seizures and coma [25]. Therefore the tendency to maximise energy distribution/dissipation emerges from those entropic measures. Taking the aforesaid perspective that the brain microstates represent configurations of "connecte" brain cell ensembles -it has been proposed that the nature of brain states are patterns of coordinated activity that can be observed as correlations/synchronization of activity in cell ensembles [25]- then the comment in the previous section on the equal distribution of energy among microstates maximising entropy finds here its neurophysiological correlate: energy, that is the neural activity, tends to be distributed as widely as possible in normal waking consciousness and the result of that energy distribution is the maximisation of brain microstates, the configurations of connectivity patterns among brain networks.

But these are not novel concepts. Scholars since times past have voiced the notion that living processes make the energy flux through the system a maximum, of course so far as compatible with constraints [41], and that the flow of energy through a system acts to organise that system [42. While these early thoughts were mostly concerned with metabolic, living systems, the idea can be adapted to the nervous system because just like metabolic pathways offer channels for energy to flow, neural communication provide further conduits for that flow. It has been proposed that the expression of this feature of maximising the energy flow/distribution in the function of the brain could be the main factor accountable for the emergence of conscious awareness. But one should always be careful about using extremal principles such as maximisation or minimisation of entropy for nonequilibrium processes [36].

Nevertheless, considering the possibility of the tendency towards maximization of energy spread could be more effective than talking about maximization of man-made metrics like entropy or information. In this regard, some scholars are reluctant to consider maximization principles as cause of processes driving living systems [43], view which may be correct because concepts like information or entropy are, in the final analysis, man-made creations developed to characterize certain phenomena; energy, on the other hand, seems to be a more fundamental aspect of natural phenomena, although here too efforts at obtaining precise and strict definitions of energy have not yielded too much success, the definitions proposed being inherently circular (see [44, chap 12]). In the final analysis, it is the gradient of energy, the energy dissipation in a final state compared to an initial state, the driving force, and the entropy is the index of that dispersal [26].

Other entropic measures used in various studies are related as well to the number of configurations of brain network connections. For example, permutation entropy applied to electroencephalographic recordings differentiates periods of epileptic seizures versus interictal activity -activity without seizures [8]-, or sample entropy applied to resting state functional neuroimaging reveals a positive correlation between high entropy and intelligence in healthy individuals [24]. Both sample entropy and permutation entropy measure the predictability of time series, the latter more concerned with the temporal ordering structure of the series, which in the end all reflect the variability in neural network connections from where the brain signals are recorded; in other words, the essence is 
again the neural collective activity patterns, or the energy distribution among brain cell ensembles. Equivalently, Shannon entropy applied to probability distributions of brain network synchrony over time -again reflecting configurations of activity or energy distribution- revealed a greater collection of functional brain connectivity motifs in the psychedelic state as compared to waking consciousness [45].

For the sake of completion, it should be noted that the ideas behind how the information flow is related to energy flows have been treated in some studies [46] and have been the subject of special issues, e.g. "Information and Energy/Matter" 47]. As well, a recent interest in considerations from the energy viewpoint has emerged, for instance in the free energy principle for biological systems 48 .

\subsection{A note on some methodological aspects and what these may reveal about brains}

It is worth mentioning that in the process of the analysis done in the aforesaid estimation of entropy associated with the number of configurations of brain network connectivity a threshold value of the phase synchronization index (derived from the analytic signal concept, see 49]) was used to determine when two signals were "connected" $[35,40]$. We will not delve into the issue of whether measuring correlations between signals (phase synchrony, coherence, cross-correlations etc.) reflect "real" connectivity, as this matter has been treated in numerous texts; suffice to say here that phase synchrony was taken as an indication of connections because when two brain networks have synchronous activity this indicates that they are probably involved in processing the same input or performing same sensorimotor action (there is a very extensive literature on anatomical and functional connectivity concepts and methods of evaluation). The synchrony threshold was taking from surrogates - that is, from random time series, or, to be more precise, the phase of the signals was randomised- derived from the original recordings. Interestingly, the average value of the phase synchrony index from the surrogate population was nearly identical to the value of the index obtained from signals in the waking state. This means, basically, that brain activity during normal wakefulness, at least in terms of synchronization, is equivalent to that of random activity (for those not conversant with what surrogates are in this type of research, let us just say in simple words that they represent random, or pseudo-random - see comments below- signals obtained from the originals and are used to assess when synchrony is "real" or just accidental). The observation that the synchrony among normal brain signals do not differ that much from that of random signals has been reported in various studies [50]; here we show a quantitative illustration: the average of the phase synchrony index in a montage of intracerebral EEG (iEEG) taken in a subject during normal waking consciousness was $0.399 \pm 0.03$, and that of the surrogate population was $0.394 \pm 0.01$, and in a monkey (a close relative) intracranial recordings (ECoG) had a synchrony index average of $0.393 \pm 0.02$, and $0.391 \pm 0.08$ for the surrogates ${ }^{\top}$. But what does this mean, for brain function and cognition in general?

First let us note that the notion of randomness is a very relative concept. To wit, number $e$ (or $\pi$ ) may be random or deterministic depending on how it is "measured". Statistical tests applied to the sequence of digits will indicate randomness. But we know how to generate those digits using a formula, in case of $e$ one formula is $e=\lim [1+1 / n]^{n}$ hence it is not random but totally determined by the mathematical expression. Hence, is $e$ random or deterministic? It depends on the viewpoint. Having said this, the fact that surrogate signals provide similar synchrony as those during waking consciousness is a consequence of the signals measured, which at the mesoscale level reflect the activity of large cellular populations, and considering that the brain has to integrate and segregate a myriad of sensorimotor transformations simultaneously in order to adequately perceive and respond to incoming stimuli $[25,51$, then this neuronal activity at the microscale level is observed at higher levels as stochastic. It does not mean a healthy brain displays random activity, rather it needs to support so many different configurations of micro-level connections (the aforesaid large entropy during conscious states) that depending on the measurement it will appear as organised or as purely stochastic; if we were to follow the activity in specific microcircuits we would see organised activity, but when the recordings sample thousands of these organised microcircuits the result is a noisy, random-like time series. Therefore, a noisy brain is a healthy brain [52. All these notions are related to the concept of critical dynamics in brain activity, commented upon in the next section.

\section{On nervous system criticality}

Before concluding this brief overview on entropy applied to neural systems, a few words on the concept of critical dynamics and how this relates to the results reviewed in this text. Basically, criticality stems from the competition

\footnotetext{
${ }^{1} \mathrm{~A}$ quick note on the phase synchrony index computation. Pairwise phase synchrony (calculated as in [49, 50] was evaluated for 46 iEEG channels and for 128 ECoG channels. Signals were pre-processed in the standard fashion and filtered using a frequency band of $28 \pm 2 \mathrm{~Hz}$. To compute the synchrony index for the surrogate population, 10 surrogates for each original signal were created and same procedure for the index computation was applied, using a 1 second running window and obtaining the average index from the total number of pairwise combinations.
} 
between the ordering tendency of the interactions among the system constituents and the disordering tendency of (stochastic) fluctuations; or, from another perspective, the redistribution of energy among the system constituents results in critical phenomena. It has been proposed that the collective organization of cellular activity in brains possess features of critical dynamics in order to mimic what is found out there in nature, which is normally described by critical dynamics [53. To be fair, let us note that there still remains debate as to whether nervous systems actually operate close to criticality -commonly assessed using power law distributions-or in another state such as oscillatory or stochastic dynamics; some studies have indicated that critical and stochastic dynamics occur at the same time in the brain [54. It has to be considered as well that features associated with criticality emerge in situations even when the dynamics are not critical, e.g. in networks with a hierarchical modular structure [55].

In any event, this criticality entails a large number of metastable states - microstates that appear and disappear- thus flexible patterns of communication are possible among brain cell ensembles that allow the nervous system to process information in an adaptive fashion: the more patterns the better to process myriads of incoming stimuli, although of course the patterns have to be organised, coordinated somehow, hence this field of study is normally referred to as brain coordination dynamics [56]. Incidentally, while the notion of instability and metastability of brain dynamics is relatively recent, one of the very early proposals of the brain as an "organ whose natural state is one of unstable equilibrium" is due to William James in 1879 [57].

It is thus adaptability the key feature that the maximisation of connectivity configurations confers the organism. As illustrations of this fact are the reports that lower entropy associated with the number of configurations of connections was found when visual input was diminished -subjects with eyes closed [35]- and a less complex network structure was manifest as compared with eyes open condition [58]. Hence the results on the maximisation of entropy mentioned in section 4 apply in reality more to the optimality of brain information processing than to consciousness, for one is very conscious when his/her eyes are closed and yet the entropy associated with network connections was lower. Can this variability of interactions among neural nets, these fluctuations in connectivity patterns, be a most fundamental organising principle of nervous system function? A direct test for this proposal requires the quantification of those fluctuations in, say, neuronal synchronization or similar observables characterising functional connections. Several results, too many to be covered here, suggest this may be the case, which led to the proposal of a possible scenario for the transition from healthy brain activity towards pathological activity 59 .

\section{Concluding remarks}

Systems tend to attain the most probable state -equilibrium, which, incidentally is a concept closely connected with that of observation time and thus somewhat relative 60]- which normally consists of a macrostate represented by the largest number of microstates. In the case of brains supporting cognition, proper brain function requires an efficient web of neural connections, which, interpreted from the perspective expounded in this text, is equivalent to saying that nervous systems tend to a widespread energy distribution among all those microstates via maximization of the number of configurations. In chemistry, for instance, one talks about the number of molecular configurations in chemical reactions; in the case of brains the microstates consist of the number of configurations of connections among neural networks; brain macrostates associated with conscious awareness possess more microstates, or configurations of connections [40], whose emergence and dissolution determine cognitive states. In other words, the embodied brains that are immersed in an environment seem to attempt to reach equilibrium, which is not possible due to the exchange of energy (information) with the environs. The intricate web of neuronal assemblies giving rise to a myriad of energy gradients results from the "neuroglial closure" in brain cell circuits [51]; when this closure, this wide interconnectedness, disappears transiently, for instance during epileptic seizures, the consequence is loss of conscious awareness. And generally speaking this is a natural tendency, just like gas molecules tend to occupy the maximal volume available, energy will redistribute in the widest manner for no particular reason but because that is the most probable outcome. The diverse natural phenomena and their specific mechanisms are details as to how the optimal energy distribution is achieved, and from these phenomena concepts of order/disorder, stochasticity/determinism, emerge.

\section{References}

[1] Stefan Thurner, Bernat Corominas-Murtra, and Rudolf Hanel. Three faces of entropy for complex systems: Information, thermodynamics, and the maximum entropy principle. Physical Review E, 96(3):32124, 2017.

[2] S P Strong, Roland Koberle, Rob R de Ruyter van Steveninck, and William Bialek. Entropy and Information in Neural Spike Trains. Physical Review Letters, 80(1):197-200, 1998. 
[3] S. Street. Upper Limit on the Thermodynamic Information Content of an Action Potential. Preprints, 2020020035, 2020.

[4] A Gomez-Mellado, T Munoz, C Paiva, Karina Vilches, and I Carvacho. Entropy production of ion thermodiffusion in cell membranes. In Journal of Physics: Conference Series, volume 1160, page 12013. IOP Publishing, 2019.

[5] Vidit Agrawal, Andrew B Cowley, Qusay Alfaori, Daniel B Larremore, Juan G Restrepo, and Woodrow L Shew. Robust entropy requires strong and balanced excitatory and inhibitory synapses. Chaos: An Interdisciplinary Journal of Nonlinear Science, 28(10):103115, 2018.

[6] Ingo C Kleppe and Hugh P C Robinson. Correlation entropy of synaptic input-output dynamics. Physical Review E, 74(4):41909, 2006.

[7] Christoph Bandt and Bernd Pompe. Permutation entropy: a natural complexity measure for time series. Physical Review Letters, 88(17):174102, 2002.

[8] Nadia Mammone, Jonas Duun-Henriksen, Troels Kjaer, and Francesco Morabito. Differentiating interictal and ictal states in childhood absence epilepsy through permutation Rényi entropy. Entropy, 17(7):4627-4643, 2015.

[9] Zhenhu Liang, Yinghua Wang, Xue Sun, Duan Li, Logan J Voss, Jamie W Sleigh, Satoshi Hagihira, and Xiaoli Li. EEG entropy measures in anesthesia. Frontiers in Computational Neuroscience, 9, feb 2015.

[10] Xiaoli Li, Gaoxian Ouyang, and Douglas A Richards. Predictability analysis of absence seizures with permutation entropy. Epilepsy research, 77(1):70-74, 2007.

[11] N Nicolaou and J Georgiou. The Use of Permutation Entropy to Characterize Sleep Electroencephalograms. Clinical $\{$ EEG $\}$ and Neuroscience, 42(1):24-28, 2011.

[12] F C Morabito, D Labate, F La Foresta, A Bramanti, G Morabito, and I Palamara. Multivariate multi-scale permutation entropy for complexity analysis of Alzheimer's disease EEG. Entropy, 14(7):1186-1202, 2012.

[13] Wajid Aziz and Muhammad Arif. Multiscale Permutation Entropy of Physiological Time Series. In 2005 Pakistan Section Multitopic Conference. IEEE, 2005.

[14] B Fadlallah, B Chen, A Keil, and J Pr \'incipe. Weighted-permutation entropy: A complexity measure for time series incorporating amplitude information. Physical Review E, 87(2):22911, feb 2013.

[15] A Vakkuri, A Yli-Hankala, P Talja, S Mustola, H Tolvanen-Laakso, T Sampson, and H Viertio-Oja. Timefrequency balanced spectral entropy as a measure of anesthetic drug effect in central nervous system during sevoflurane, propofol, and thiopental anesthesia. Acta Anaesthesiologica Scandinavica, 48(2):145-153, feb 2004.

[16] Xiaoli Li, Duan Li, Zhenhu Liang, Logan J Voss, and Jamie W Sleigh. Analysis of depth of anesthesia with Hilbert-Huang spectral entropy. Clinical Neurophysiology, 119(11):2465-2475, nov 2008.

[17] O A Rosso, S Blanco, J Yordanova, V Kolev, A Figliola, M Schürmann, and E Ba \csar. Wavelet entropy: a new tool for analysis of short duration brain electrical signals. Journal of neuroscience methods, 105(1):65-75, 2001.

[18] Jürgen Fell, Joachim Röschke, Klaus Mann, and Cornelius Schäffner. Discrimination of sleep stages: a comparison between spectral and nonlinear \{EEG\} measures. Electroencephalography and Clinical Neurophysiology, 98(5):401-410, may 1996.

[19] N Kannathal, Min Lim Choo, U Rajendra Acharya, and P K Sadasivan. Entropies for detection of epilepsy in EEG. Computer methods and programs in biomedicine, 80(3):187-194, 2005.

[20] R Quian Quiroga, O A Rosso, E Ba?ar, and M Schrmann. Wavelet entropy in event-related potentials: a new method shows ordering of \{EEG\} oscillations. Biological Cybernetics, 84(4):291-299, mar 2001.

[21] Hasan A Al-Nashash, Joseph S Paul, Wendy C Ziai, Daniel F Hanley, and Nitish V Thakor. Wavelet Entropy for Subband Segmentation of EEG During Injury and Recovery. Annals of Biomedical Engineering, 31(6):653-658, jun 2003. 
[22] Christos A Frantzidis, Ana B Vivas, Anthoula Tsolaki, Manousos A Klados, Magda Tsolaki, and Panagiotis D Bamidis. Functional disorganization of small-world brain networks in mild Alzheimer's Disease and amnestic Mild Cognitive Impairment: an EEG study using Relative Wavelet Entropy (RWE). Frontiers in Aging Neuroscience, 6:224, 2014.

[23] Y Yao, W L Lu, B Xu, C B Li, C P Lin, D Waxman, and J F Feng. The Increase of the Functional Entropy of the Human Brain with Age. Scientific Reports, 3(1), oct 2013.

[24] Glenn N Saxe, Daniel Calderone, and Leah J Morales. Brain entropy and human intelligence: A resting-state fMRI study. PloS one, 13(2), 2018.

[25] Jose Luis Perez Velazquez, Diego Martin Mateos, and Ramon Guevara Erra. On a simple general principle of brain organization. Frontiers in Neuroscience, 13:1106, 2019.

[26] Frank L Lambert. Disorder-A cracked crutch for supporting entropy discussions. Journal of Chemical Education, 79(2):187, 2002.

[27] Moses O Sokunbi, Wilson Fung, Vijay Sawlani, Sabine Choppin, David E J Linden, and Johannes Thome. Resting state fMRI entropy probes complexity of brain activity in adults with ADHD. Psychiatry Research: Neuroimaging, 214(3):341-348, 2013.

[28] Moses O. Sokunbi, Victoria B. Gradin, Gordon D. Waiter, George G. Cameron, Trevor S. Ahearn, Alison D. Murray, Douglas J. Steele, and Roger T. Staff. Correction: Nonlinear Complexity Analysis of Brain fMRI Signals in Schizophrenia. PloS one, 9(8):e105741, 2014.

[29] William Bialek. Biophysics: searching for principles. Princeton University Press, 2012.

[30] Gualtiero Piccinini and Andrea Scarantino. Information processing, computation, and cognition. Journal of biological physics, 37(1):1-38, 2011.

[31] Fred Rieke, David Warland, Rob R de Ruyter van Steveninck, and William Bialek. Spikes: Exploring the Neural Code. Press, MIT, 1999.

[32] Peter Sterling and Simon Laughlin. Principles of Neural Design. The \{MIT\} Press, jun 2015.

[33] Jonathan D Victor. Approaches to Information-Theoretic Analysis of Neural Activity. Biological Theory, $1(3): 302-316$, sep 2006.

[34] Alexander Borst and Frédéric E Theunissen. Information theory and neural coding. Nature neuroscience, 2(11):947-957, 1999.

[35] R Guevara Erra, D M Mateos, R Wennberg, and J L Perez Velazquez. Statistical mechanics of consciousness: Maximization of information content of network is associated with conscious awareness. Physical Review E, $94(5): 52402,2016$.

[36] Leonid M Martyushev and Vladimir D Seleznev. Maximum entropy production principle in physics, chemistry and biology. Physics reports, 426(1):1-45, 2006.

[37] Myron Tribus and Edward C McIrvine. Energy and information. Scientific American, 225(3):179-190, 1971.

[38] Oliver Sacks. An anthropologist on Mars: Seven paradoxical tales. Vintage, 2012.

[39] Edmund Jack Ambrose. The nature and origin of the biological world. Technical report, 1982.

[40] D M Mateos, R Wennberg, R Guevara, and J L Perez Velazquez. Consciousness as a global property of brain dynamic activity. Physical Review E, 96(6):62410, 2017.

[41] Alfred J Lotka. Contribution to the energetics of evolution. Proceedings of the National academy of Sciences of the United States of America, 8(6):147, 1922.

[42] Harold Morowitz and Eric Smith. Energy flow and the organization of life. Complexity, 13(1):51-59, 2007.

[43] Hermann Haken. Information and self-organization: A macroscopic approach to complex systems. Springer Science \& Business Media, 2006.

[44] Paul L Nunez. Brain, mind, and the structure of reality. Oxford University Press, 2012. 
[45] Robin Lester Carhart-Harris, Robert Leech, Peter John Hellyer, Murray Shanahan, Amanda Feilding, Enzo Tagliazucchi, Dante R Chialvo, and David Nutt. The entropic brain: a theory of conscious states informed by neuroimaging research with psychedelic drugs. Frontiers in human neuroscience, 8:20, 2014.

[46] Pedro C Marijuán, Jorge Navarro, and Raquel del Moral. How prokaryotes 'encode'their environment: Systemic tools for organizing the information flow. Biosystems, 164:26-38, 2018.

[47] Gordana Dodig Crnkovic. Information and energy/matter, 2012.

[48] Friston Karl. A free energy principle for biological systems. Entropy, 14(11):2100-2121, 2012.

[49] F Mormann, K Lehnertz, P David, and C E Elger. Mean phase coherence as a measure for phase synchronization and its application to the EEG of epilepsy patients. Physica D: Nonlinear Phenomena, 144(3):358-369, 2000 .

[50] Luis Garcia Dominguez, Richard A Wennberg, William Gaetz, Douglas Cheyne, O Carter Snead, and Jose Luis Perez Velazquez. Enhanced synchrony in epileptiform activity? Local versus distant phase synchronization in generalized seizures. Journal of neuroscience, 25(35):8077-8084, 2005.

[51] Jose Luis Perez Velazquez. On the emergence of cognition: from catalytic closure to neuroglial closure. Journal of Biological Physics, pages 1-25, 2020.

[52] Andrea B Protzner, Taufik Valiante, Natasa Kovacevic, Cornelia McCormick, and Mary Pat McAndrews. Hippocampal signal complexity in mesial temporal lobe epilepsy: a noisy brain is a healthy brain. Archives italiennes de biologie, 148(3):289-297, 2010.

[53] D R Chialvo. Emergent complex neural dynamics. Nature physics, 6(10):744-750, 2010.

[54] Jesse Tinker and Jose Luis Perez Velazquez. Power law scaling in synchronization of brain signals depends on cognitive load. Frontiers in Systems Neuroscience, 8, may 2014.

[55] Eric J Friedman and Adam S Landsberg. Hierarchical networks, power laws, and neuronal avalanches. Chaos: An Interdisciplinary Journal of Nonlinear Science, 23(1):13135, 2013.

[56] J A Scott Kelso. Dynamic patterns: The self-organization of brain and behavior. MIT press, 1995.

[57] W M JAMES. I. - ARE WE AUTOMATA? Mind, os-4(13):1-22, 1879.

[58] Marie-Therese Horstmann, Stephan Bialonski, Nina Noennig, Heinke Mai, Jens Prusseit, Jörg Wellmer, Hermann Hinrichs, and Klaus Lehnertz. State dependent properties of epileptic brain networks: Comparative graph-theoretical analyses of simultaneously recorded EEG and MEG. Clinical Neurophysiology, 121(2):172$185,2010$.

[59] Jose Luis Perez Velazquez and Marina Frantseva. The Brain-Behavior Continuum: The Subtle Transition Between Sanity and Insanity. World Scientific, 2011.

[60] Craig Callender. Taking thermodynamics too seriously. Studies in history and philosophy of science part B: studies in history and philosophy of modern physics, 32(4):539-553, 2001. 